\title{
Relationship between the extent of deep venous thrombosis and the extent of acute pulmonary embolism as assessed by CT angiography
}

\author{
${ }^{1} \mathrm{~B}$ GHAYE, MD, ${ }^{1} \mathrm{~V}$ WILLEMS, MD, ${ }^{1} \mathrm{~A}$ NCHIMI, MD, ${ }^{1} \mathrm{~L}$ KOUOKAM, MD, ${ }^{1} \mathrm{C}$ NOUKOUA, MD, ${ }^{3} \mathrm{~V}$ DE \\ MAERTELAER, PhD, ${ }^{2} \mathrm{P}$ A GEVENOIS, MD, PhD and ${ }^{1} \mathrm{R} F$ DONDELINGER, MD
}

\begin{abstract}
${ }^{1}$ Department of Medical Imaging, University Hospital of Liege, Liege, ${ }^{2}$ Department of Medical Imaging, Erasme Hospital, Université libre de Bruxelles and ${ }^{3}$ Statistical Unit, Institute of Interdisciplinary Research in Human and Molecular Biology, Université libre de Bruxelles, Belgium
\end{abstract}

\begin{abstract}
The aim of our study is to investigate prospectively the quantitative relationship between deep venous thrombosis (DVT) and acute pulmonary embolism (PE). 110 patients clinically suspected of having venous thromboembolic disease underwent combined CT pulmonary angiography (CTPA) and venography of lower limb veins. 44 patients presented with clinical signs of DVT and positive ultrasonography or ascending venography, but no clinical sign of PE (Group 1). 66 patients presented with clinical signs of PE and positive CTPA (Group 2). Clot load in lower limb veins and pulmonary arteries were scored by two independent readers, each using two separate systems for DVT and two for PE. 27 (61\%) patients in Group 1 also had PE, and 55 (83\%) patients in Group 2 also had DVT. Correlations between PE and DVT scores were weak but statistically significant in Group 2 ( $r_{\mathrm{s}}$ ranging from $\left.0.470-0.520 ; p \leq 0.001\right)$, but only some were significant in Group 1 ( $r_{\mathrm{s}}$ ranging from $0.253-0.318 ; p$-values ranging from $0.035-0.097)$. In conclusion, although PE occurs in a majority of patients with DVT, and vice versa, the amount/burden of clot load in one condition does not necessarily indicate — or indicates only weakly — the degree of burden in the other condition.
\end{abstract}

Received 3 December 2007 Revised 24 January 2008 Accepted 11 February 2008

DOI: $10.1259 / \mathrm{bjr} / 54005160$

(C) 2009 The British Institute of Radiology
Venous thromboembolic disease comprises pulmonary embolism (PE) and deep venous thrombosis (DVT) [1-12]. A patient may present with either of these conditions, or both, and may not always exhibit the signs of one condition. This is particularly concerning with silent PE, where the risk of death increases with the presence of coexisting pulmonary disease, right heart failure to compensate for pulmonary hypertension, and embolic recurrences (which come from the lower limb veins in $90 \%$ of cases, and which further increase pulmonary arterial obstruction) [13]. In patients clinically suspected of having PE, two imaging approaches have been recommended: investigating firstly the lower limb veins $[8,9,11]$ or the pulmonary arteries $[3,5,10,14]$. If DVT or $\mathrm{PE}$ is found with either investigation, the presence of the alternative condition is often simply assumed and no further investigation is conducted. Patients are then treated with anticoagulation therapy in order to prevent growth of DVT and/or PE recurrence.

Because venous thromboembolic disease can be dynamic, treatment must be carefully matched to the extent of the condition, and adapted if necessary. As pointed out by several investigators, thrombotic disease can be treated inappropriately: silent PE can go unnoticed and, as a consequence, can be treated insufficiently or, when mistaken to have progressed from ineffective DVT

Address correspondence to: Benoit Ghaye, Department of Medical Imaging, University Hospital Sart Tilman - B35, B - 4000 Liege, Belgium. E-mail: bghaye@chu.ulg.ac.be treatment, can result in an overly aggressive or unnecessary change in treatment [2,6,15-17]. Girard et al [4] have shown that the probability of PE is closely correlated to the extent of DVT in lower limb veins, and the greater the extent of PE, the higher the probability of residual DVT. However, it is not known whether the actual load of the clot in lower limb veins could predict clot load in pulmonary arteries, and vice versa. The aim of our study, therefore, was to investigate prospectively the qualitative and quantitative relationship between DVT and acute PE.

\section{Methods and patients}

\section{Patients}

The study protocol was approved by the Ethics Committee of our hospital and oral informed consent was obtained from all patients.

In order to investigate the relationship between the clot load in the lower limb veins and the clot load in the pulmonary arteries, we created two groups of patients, which reflect the population seen in the clinical setting: (i) patients with DVT who were not suspected of having PE by their physicians; and (ii) patients who were suspected of PE - with or without suspicion of DVT - by their physicians. Patients from Group 1 were selected as follows: 481 consecutive patients clinically suspected of DVT were referred to our department for an imaging examination of their lower limb veins either by ultrasound 
$(n=464)$ or ascending venography $(n=17)$. From these 481 patients, 72 had a DVT, whereas 44 of these "DVT patients" were not clinically suspected of acute PE. These 44 patients (22 (50\%) men and 22 (50\%) women; mean ( \pm standard deviation) age, $61 \pm 15$ years) became Group 1 (i.e. patients with confirmed DVT). For comparison, patients from Group 2 were selected as follows: 384 consecutive patients from the same time period with suspected PE underwent CT pulmonary angiography (CTPA) and CT venography prospectively, resulting in 66 patients (35 (53\%) men and $31(47 \%)$ women; mean age, $60 \pm 17$ years) with confirmed PE on CTPA who became Group 2 (i.e. patients with confirmed PE). As such confirmation could not be provided by indeterminate CTPA, 29 patients with such an examination were not included in our study group. As PE is the condition of greater concern in clinical practice, we did not insist that Group 2 patients show signs of DVT. There was thus no overlap between both groups of patients.

\section{CT examination}

All patients underwent combined CTPA and CT venography of the lower limbs. Group 1 patients were included within $6 \mathrm{~h}$ of referral to the centre. All CT examinations were performed on a commercially available helical scanner (PQ 5000; Philips Medical Systems, Eindhoven, the Netherlands) with the same acquisition parameters. Patients were examined while in the supine position. Before CT examination, they were trained to breath-hold for 20-40 s after full inspiration. $20 \mathrm{~s}$ before CT acquisition, intravenous injection of $140 \mathrm{ml}$ of $30 \%$ iodinated contrast medium (Xenetix ${ }^{\circledR}$; Guerbet, Aulnay-sousBois, France) was initiated at a flow rate of $3 \mathrm{~cm}^{3} \mathrm{~s}^{-1}$. A caudo-cranial acquisition was performed with $2 \mathrm{~mm}$ collimation, $1 \mathrm{~s}$ rotation time, a pitch of 2:1 at $120 \mathrm{~mA}$ and $130 \mathrm{kVp}$. This acquisition started $2 \mathrm{~cm}$ below the top of the diaphragm and ended at the upper aspect of the aortic arch, enabling visualization of the heart and pulmonary arteries up to the subsegmental branching order. Scans were reconstructed at $1 \mathrm{~mm}$ intervals with a soft-tissue algorithm. CT venography was obtained $210 \mathrm{~s}$ after the start of injection, from the calf to diaphragm, using a sequential acquisition of $5 \mathrm{~mm}$ thick slices at $20 \mathrm{~mm}$ intervals, $1 \mathrm{~s}$ rotation time at $100-125 \mathrm{~mA}$ and $130 \mathrm{kVp}$. The effective radiation dose delivered with these settings was computer simulated with commercially available software (CT Expo ${ }^{\circledR}$; University of Hannover, Hannover, Germany; Version 1.5, 2005). The corresponding calculated radiation dose was $2.7 \mathrm{mSv}$ and $2.9 \mathrm{mSv}$ in women and $2.3 \mathrm{mSv}$ and $2.4 \mathrm{mSv}$ in men for CTPA and CT venography, respectively. All images were read immediately by the senior radiologist conducting the examination. The results were then reported to the referring clinician, who integrated them into the final case management decision. This interpretation was not taken into consideration for the present study.

\section{Image analysis}

For the purpose of this study, each CT examination was reviewed on a workstation (Voxel Q; Philips
Medical Systems, Eindhoven, the Netherlands) by two independent readers, who were asked to judge whether its quality was sufficient for scoring PE and DVT and, if sufficient for both, to score the clot load in the pulmonary arteries and lower limb veins. Reader 1 was a chest radiologist with 12 years of experience in reading thoracic CT scans. Reader 2 was a 5-year radiology resident who had 3 years' experience in reading CTPA. The readers were blinded to clinical data and results of other imaging techniques, but they knew that patients were suspected to have PE or DVT but not to which group they belonged.

In pulmonary arteries, the clot load was scored according to Mastora et al [18] and Qanadli et al [19]. The scoring system proposed by Mastora et al [18] is based on the number of arteries involved up to the segmental branching order and the degree of lumen narrowing. The scoring system proposed by Qanadli et al [19] is based on these criteria as well, but also gives weight to the branching order of the involved arteries (i.e. the more proximal, the more severe). In lower limb veins, the clot load was scored according to Björgell et al [20] and Ouriel et al [21]. The scoring system proposed by Björgell et al [20] is based on the number of involved veins and the length of obstruction. The scoring system proposed by Ouriel et al [21] also considers the number of veins involved, but includes the size of the vein as well. The four scoring systems are detailed in Appendix A.

For further analyses, the clot load score in pulmonary arteries and lower limb veins was expressed as a percentage. This was calculated by dividing the score obtained with each system by the maximum possible score for the considered system and multiplying by 100 .

\section{Statistical analysis}

Differences in score values between groups and between readers were tested with Mann-Whitney tests. The degree of association between scores was investigated with Spearman's correlation coefficients. The associated $p$-values are obtained after testing the null hypotheses of absence of correlation. Statistical significance for all tests was set at a $p$-value of $<0.05$. The statistical software used was SPSS for Windows (Release 13; SPSS, Chicago, IL).

\section{Results}

The quality of all examinations was judged sufficient for scoring both PE and DVT. Among 44 patients who constituted Group 1, each reader identified the same 27 $(61 \%)$ patients with unsuspected PE at CTPA. Among the 66 patients of Group 2, each reader identified the same 55 (83\%) patients with DVT at CT venography.

Scores of PE and DVT are summarized in Table 1. For both groups of patients, no statistical difference was detected between readers. Patients in Group 1 had a PE clot load significantly lower than those in Group 2 $(p<0.001)$, whereas patients in Group 1 had a DVT clot load significantly higher than those in Group 2 ( $p=0.001-$ 0.019). 
Table 1. Comparison between clot load scores in pulmonary arteries and lower limb veins

\begin{tabular}{|c|c|c|c|c|c|c|c|c|c|}
\hline & \multirow[b]{2}{*}{$\begin{array}{l}\text { Scoring } \\
\text { system }\end{array}$} & \multicolumn{3}{|c|}{ Group $1(n=44)$} & \multicolumn{3}{|c|}{ Group $2(n=66)$} & \multicolumn{2}{|c|}{$\begin{array}{l}\text { Differences between } \\
\text { Groups ( } p \text {-value) }\end{array}$} \\
\hline & & Reader 1 & Reader 2 & $\begin{array}{l}\text { Difference } \\
\text { between } \\
\text { Readers } \\
\text { ( } p \text {-value) }\end{array}$ & Reader 1 & Reader 2 & $\begin{array}{l}\text { Differences } \\
\text { between } \\
\text { Readers } \\
\text { (p-value) }\end{array}$ & Reader 1 & Reader 2 \\
\hline \multirow{2}{*}{ PE clot load (\%) } & $\begin{array}{l}\text { Qanadli et } \\
\text { al [19] }\end{array}$ & $3.3 \pm 0.8$ & $3.4 \pm 0.7$ & 0.606 & $10.2 \pm 1.1$ & $10.5 \pm 1.2$ & 0.226 & $<0.001$ & $<0.001$ \\
\hline & $\begin{array}{l}\text { Mastora et } \\
\text { al [18] }\end{array}$ & $7.9 \pm 2.0$ & $9.0 \pm 2.4$ & 0.895 & $34.3 \pm 4.4$ & $35.1 \pm 4.4$ & 0.266 & $<0.001$ & $<0.001$ \\
\hline \multirow{2}{*}{ DVT clot load (\%) } & $\begin{array}{l}\text { Björgell et } \\
\text { al [20] }\end{array}$ & $8.9 \pm 1.0$ & $9.2 \pm 1.0$ & 0.648 & $5.5 \pm 0.7$ & $5.1 \pm 0.6$ & 0.110 & 0.007 & 0.001 \\
\hline & $\begin{array}{l}\text { Ouriel et al } \\
\text { [21] }\end{array}$ & $10.8 \pm 1.8$ & $11.7 \pm 1.7$ & 0.138 & $5.8 \pm 0.9$ & $6.0 \pm 0.8$ & 0.076 & 0.019 & 0.010 \\
\hline
\end{tabular}

Data given as the mean \pm standard error of the mean. PE, pulmonary embolism; DVT, deep venous thrombosis.

Among patients in Group 2, 22 (33\%) had clinical findings suggestive of coexisting DVT (as reported by the physician in charge of the patient). Although clot load was somewhat higher in these patients than in those without these clinical findings, it was not significant, regardless of the reader $(p=0.077-0.477)$.

Spearman's correlation coefficient between clot load scores of PE assessed according to Mastora et al [18] and Qanadli et al [19] was $0.983(p<0.001)$. Clot load scores of DVT assessed according to Björgell et al [20] and Ouriel et al [21] resulted in a correlation coefficient of 0.906 $(p<0.001)$.

Spearman's correlation coefficients between clot load scores of PE and DVT are listed in Table 2. These coefficients indicate significant correlations in Group 2. Within Group 1, however, results were mixed: some correlations were not significant and those that were, were weak.

\section{Discussion}

This study demonstrates that: (i) two-thirds of our patients presenting with DVT did have a concomitant but clinically unsuspected PE; (ii) four-fifths of our patients with PE also had concomitant DVT; (iii) PE clot load is higher in patients referred for clinically suspected PE than in those referred for clinically suspected DVT and, conversely, DVT clot load is higher in patients clinically suspected of DVT than in those clinically suspected of PE; (iv) depending on the clinical presentation and the scoring system used, the relationship between clot loads in pulmonary arteries and lower limb veins is either not significant, or significant but weak; and (v) results between two scoring systems assessing the clot load in lower limb veins and between those assessing pulmonary arteries are highly correlated, regardless of the reader.

Our study confirms that, regardless of the clinical condition that reveals venous thromboembolic disease, PE and DVT very frequently coexist. This suggests that the presence of one condition of the disease should be inferred when the presence of the other is confirmed. Indeed, in patients who had DVT, we found a $61 \%$ prevalence of clinically unsuspected $\mathrm{PE}$. In patients who had a clinically suspected PE (confirmed by CTPA), the prevalence of coexisting DVT reached $83 \%$. All of our patients had an examination quality adequate to score pulmonary arteries. This may be different from other studies and could be explained by our recruitment process: patients in Group 1 had no dyspnoea or pulmonary symptoms and those in Group 2 had positive CTPA. However, our results are in line with previous studies that have reported clinically unsuspected PE in $34-58 \%$ of patients with acute DVT, regardless of the imaging technique used $[2,6,15-17,22-26]$, and a prevalence of DVT in $72-82 \%$ of patients with clinically suspected/confirmed PE [1, 4, 27]. The relationship between the location of the upper end of the venous clot in the lower limbs and the frequency of associated PE, however, remains controversial. Some authors have reported that the risk of PE is higher for proximal DVT than for distal DVT $[22,23]$, but others have not reported such a relationship [6, 24-26].

Our study shows that PE clot load is higher in patients referred for clinical suspicion of PE than in those referred

Table 2. Correlation between PE and DVT clot load scores

\begin{tabular}{llll}
\hline & & Qanadli et al [19] & Mastora et al [18] \\
\hline \multirow{2}{*}{ Group 1 $(n=44)$} & Ouriel et al [21] & $r_{\mathrm{s}}=0.318$ & $r_{\mathrm{s}}=0.316$ \\
& & $p=0.035$ & $p=0.037$ \\
& Björgell et al [20] & $r_{\mathrm{s}}=0.264$ & $r_{\mathrm{s}}=0.253$ \\
& & $p=0.083$ & $p=0.097$ \\
Group 2 $(n=66)$ & Ouriel et al [21] & $r_{\mathrm{s}}=0.517$ & $r_{\mathrm{s}}=0.520$ \\
& & $p<0.001$ & $p<0.001$ \\
& Björgell et al [20] & $r_{\mathrm{s}}=0.454$ & $r_{\mathrm{s}}=0.470$ \\
& & $p=0.001$ & $p<0.001$ \\
\hline
\end{tabular}

PE, pulmonary embolism; DVT, deep venous thrombosis; $r_{\mathrm{s}}$, Spearman's correlation coefficient. 
for clinical suspicion of DVT. Conversely, in patients presenting with a clinical suspicion of DVT, DVT clot load is higher than in those presenting with clinical suspicion of PE. Our results are in accordance with those of previous studies that have investigated either DVT or PE separately. Björgell et al [28] have shown higher DVT clot loads in patients presenting with symptoms of DVT compared with those without symptoms. Conversely, studies have shown relationships between PE clot load and clinical severity of PE [29-31].

Furthermore, our study reveals the weakness of the relationship between clot load scores for PE and DVT. This relationship is indeed weak and, depending on the clinical presentation and the scoring system used, is often not even significant. With highest correlation coefficients approximating 0.500, our results should invite clinicians to be extremely cautious when inferring the clot load of PE to DVT, and vice versa. Studies using either qualitative or quantitative parameters for each condition of thromboembolic disease also described the absence of any such relationship. Indeed, Girard et al [4] reported no relationship between the Miller score and the anatomical level of the upper end of the clot in lower limb veins. Similarly, Lopez-Beret et al [6] found that, in patients with proximal DVT (i.e. within the iliofemoral and/or femoropopliteal veins), the number of pulmonary segments affected by PE is not any higher than in patients with more distal DVT. Gouzien et al [16] also found no relationship between the branching order of pulmonary arteries with thrombus on CTPA and the anatomical level of DVT on ultrasound. Therefore, even if the hypothesis that large pulmonary arteries are more likely to be obstructed by emboli originating from large (i.e. proximal) veins makes intuitive sense, it has not been confirmed by our study or any other study that we are aware of. We found that the PE clot load score is only weakly linked to the DVT clot load score, meaning that patients with a low PE clot load (i.e. clots in peripheral pulmonary arteries) may have a high DVT clot load score (i.e. clots in proximal lower limb veins) and thus be at high risk of PE recurrence [21]. Similarly, a limited DVT might actually be the small remnant of a previously extensive thrombus, the bulk of which may have migrated into the pulmonary arteries [24]. This is an important result as, although the detection of pulmonary emboli with CT pulmonary angiography may be an important indicator of concomitant DVT, it cannot predict the extent of the underlying DVT, which potentially heralds a more severe embolic event. This is also of importance in the controversial therapeutic decision to treat patients having isolated sub-segmental $\mathrm{PE}$, as some will also have remaining clot burden in lower limbs veins that may migrate into the pulmonary arteries.

We have used four scoring systems: two for lower limb veins and two for pulmonary arteries. We selected these systems because our study required a complete assessment of both conditions of venous thromboembolic disease. The system proposed by Ouriel et al [21] has been designed to assess clot load in lower limb veins by calculating a volumetric index in 14 venous segments. The system proposed by Björgell et al [20] has been designed for venography, ultrasound, CT and MRI, and considers 12 venous segments. The two systems we chose for pulmonary arteries were specifically designed for cross-sectional imaging of PE. Qanadli et al [19] have proposed an index that differentiates between partial or complete obstruction of each pulmonary artery segment. These authors have reported good reproducibility and strong correlation between this score and Miller's pulmonary angiography index. Mastora et al [18] have proposed a system that complements this; by assessing the obstruction of each pulmonary vessel on a five-point scale, it provides information on the perfusion of the vessels distal to the thrombus. These authors have also reported a relationship between this score and echocardiographic findings. The strong correlations observed in our study (regardless of the reader) between the systems used for PE and between the ones used for DVT suggest that either of the two systems could be used without reservation.

Our study has certain limitations. Firstly, the scoring of DVT was based on indirect CT venography obtained through sequential acquisition. When compared with helical acquisition of contiguous CT sections, limited DVT could, in principle, have been missed. However, the risk of missing a limited DVT was reduced by the very small $15 \mathrm{~mm}$ increments between each CT section [32]. Secondly, the system proposed by Ouriel et al [21], which was primarily designed for conventional venography, was adapted for CT. However, the system proposed by Björgell et al [20] required no adaptation because it is designed for both conventional venography and crosssectional imaging. The strong correlations observed between results obtained with both systems suggest that the adaptation we made did not bias our results. Thirdly, PE was scored from images obtained with a singledetector row helical CT scanner with $2 \mathrm{~mm}$ collimation. This collimation is adequate to evaluate PE down to the segmental branching order [33]. This would not affect the scoring system of Mastora et al [18], which considers the segmental pulmonary arteries, but may have influenced the system of Qanadli et al [19], which considers subsegmental arteries. However, the weight of subsegmental pulmonary arteries in this system is only marginal. In addition, because we observed strong correlations between both scoring systems for PE, a possible systematic bias flawing our results is highly unlikely. Conversely, we might speculate that submillimetre collimation at multidetector row CT might increase the number of clinically unsuspected PEs in patients with confirmed DVT, and would even reinforce our conclusion that PE and DVT coexist in the vast majority of patients. Fourthly, the design of our study prevented us from estimating the pre-test probability of PE and DVT. Scoring systems for estimating pre-test probability were not implemented in our institution at the time of this study. In addition, as patients in Group 1 were not suspected of PE and one-half of patients in Group 2 were not suspected of DVT, systems such as those proposed by Wells et al for PE [3] and for DVT [34] would not have been applicable. Fifth, as several statistical tests were performed, we could have adjusted for multiple testing by lowering the 0.05 cut-off $p$-value that was used to indicate statistical significance. Nevertheless, had we applied the very conservative Bonferroni correction [35], our conclusions would remain the same. Correlations between clot load scores of PE 
and clot load scores of DVT would remain significant in Group 2, and would have become even weaker in Group 1.

In conclusion, this study shows that, although PE occurs in the majority of patients with confirmed DVT, and vice versa, the extent of PE can not be assumed from the extent of DVT. It is also important to recognize that PE load cannot predict DVT load, as further management strategies (e.g. inferior vena cava filter) may be required for the treatment of DVT.

\section{Appendix A}

\section{Pulmonary artery clot load scores}

\section{Mastora et al [18]}

The score includes 5 mediastinal arteries (pulmonary artery trunk, right and left pulmonary arteries, and right and left interlobar arteries), 6 lobar arteries and 20 segmental pulmonary arteries ( 3 in the upper lobes, 2 in the middle lobe and the lingula, and 5 in the lower lobes). The score is based on the percentage of obstructed surface of each central and peripheral pulmonary artery section, using a 5-point scale $(1,<25 \% ; 2,25-49 \% ; 3,50-$ $74 \% ; 4,75-99 \%$; and 5, 100\%). A central score (mediastinal and lobar arteries), a peripheral score (segmental arteries) and a global score (central and peripheral pulmonary arteries) are calculated. The maximum score is 155 .

\section{Qanadli et al [19]}

The arterial tree of each lung is regarded as having 10 segmental arteries ( 3 in the upper lobes, 2 in the middle lobe and the lingula, and 5 in the lower lobes). The presence of one embolus in a segmental artery is scored as 1 point, and an embolus in a more proximal artery is scored according to the number of segmental arteries arising from this artery. To provide additional information about the residual perfusion distal to the embolus, a weighting factor is used for each value ( 0 , no defect; 1 , partial occlusion; and 2, complete occlusion). The maximum obstruction score is 40. An isolated subsegmental embolus is considered as a partially occluded segmental artery and assigned a value of 1 .

\section{Lower limb veins clot load scores}

\section{Björgell et al [20]}

This score is designed to be applicable to venography, ultrasound, CT and MRI. 12 vein segments are considered: inferior vena cava, common iliac veins, external iliac veins, common femoral veins, superficial femoral veins, deep femoral veins, popliteal veins, gastrocnemius veins, anterior tibial veins, posterior tibial veins, peroneal veins and sural veins. The relative length of the thrombus in each segment is scored from 0 to 3 . The maximum score is 36 per limb.

\section{Ouriel et al [21]}

14 venous segments are considered: inferior vena cava, common iliac veins, external iliac veins, internal iliac veins, common femoral veins, superficial femoral veins, deep femoral veins, popliteal veins, anterior tibial veins, posterior tibial veins and peroneal veins. A normalized volumetric score is calculated for each segment by combining measurements of $\mathrm{CT}$, ultrasound and venography. Partially occluded veins are assigned a score of one half of the score value for this segment. The score ranges from 1 (for a single calf vein) to 26 (for the inferior vena cava). The maximum score is 63 per limb.

\section{Acknowledgments}

The authors thank Denis Tack, MD, PhD, Department of Medical Imaging, Erasme Hospital, Université libre de Bruxelles, Belgium, for his help in the preparation of the manuscript.

\section{References}

1. Hull RD, Hirsh J, Carter CJ, Jay RM, Dodd PE, Ockelford PA, et al. Pulmonary angiography, ventilation lung scanning, and venography for clinically suspected pulmonary embolism with abnormal perfusion lung scan. Ann Intern Med 1983;98:891-9.

2. Moser KM, Fedullo PF, LitteJohn JK, Crawford R. Frequent asymptomatic pulmonary embolism in patients with deep venous thrombosis. JAMA 271:223-5.

3. Wells PS, Ginsberg JS, Anderson DR, Kearon C, Gent M, Turpie AG, et al. Use of a clinical model for safe management of patients with suspected pulmonary embolism. Ann Intern Med 1998;129:997-1005.

4. Girard P, Musset D, Parent F, Maitre S, Phlippoteau C, Simonneau G. High prevalence of detectable deep venous thrombosis in patients with acute pulmonary embolism. Chest 1999;116:903-8.

5. Tapson VF, Carroll BA, Davidson BL, Elliott CG, Fedullo $\mathrm{PF}$, Hales CA, et al. The diagnostic approach to acute venous thromboembolism. Clinical practice guideline. American Thoracic Society. Am J Respir Crit Care Med 1999;160:1043-66.

6. Lopez-Beret P, Pinto JM, Romero A, Orgaz A, Fontcuberta J, Oblas M. Systematic study of occult pulmonary thromboembolism in patients with deep venous thrombosis. J Vasc Surg 2001;33:515-21.

7. Borst-Krafek B, Fink AM, Lipp C, Umek H, Kohn H, Steiner A. Proximal extent of pelvic vein thrombosis and its association with pulmonary embolism. J Vasc Surg 2003 37:518-22.

8. Schoepf UJ, Costello P. CT angiography for diagnosis of pulmonary embolism: state of the art. Radiology 2004;230:329-37.

9. Perrier A, Roy PM, Aujesky D, Chagnon I, Howarth N, Gourdier AL, et al. Diagnosing pulmonary embolism in outpatients with clinical assessment, D-dimer measurement, venous ultrasound, and helical computed tomography: a multicenter management study. Am J Med 2004;116:291-9.

10. Wildberger JE, Mahnken AH, Das M, Kuttner A, Lell M, Gunther RW. CT imaging in acute pulmonary embolism: diagnostic strategies. Eur Radiol 2005;15:919-29.

11. Elias A, Cazanave A, Elias M, Chabbert V, Juchet $H$, Paradis $\mathrm{H}$, et al. Diagnostic management of pulmonary embolism using clinical assessment, plasma D-dimer assay, complete lower limb venous ultrasound and helical computed 
tomography of pulmonary arteries. A multicentre clinical outcome study. Thromb Haemost 2005;93:982-8.

12. Perrier A, Roy PM, Sanchez O, Le Gal G, Meyer G, Gourdier $\mathrm{AL}$, et al. Multidetector-row computed tomography in suspected pulmonary embolism. $\mathrm{N}$ Engl J Med 2005;352:1760-8.

13. Wood KE. Major pulmonary embolism: review of a pathophysiologic approach to the golden hour of hemodynamically significant pulmonary embolism. Chest 2002;121:877-905.

14. Fedullo PF, Tapson VF. Clinical practice. The evaluation of suspected pulmonary embolism. $\mathrm{N}$ Engl J Med 2003;349:1247-56.

15. Huisman MV, Buller HR, ten Cate JW, van Royen EA, Vreeken J, Kersten MJ, et al. Unexpected high prevalence of silent pulmonary embolism in patients with deep venous thrombosis. Chest 1989;95:498-502.

16. Gouzien P, Chabierski M, Baccialone J, Jeanbourquin D. Asymptomatic pulmonary embolism and venous thrombosis of the lower limbs. Study with spiral X-ray computed tomography. J Radiol 1996;77:125-8.

17. Monreal M, Ruiz J, Fraile M, Bonet M, Davant E, Muchart J, Vallejos V. Prospective study on the usefulness of lung scan in patients with deep vein thrombosis of the lower limbs. Thromb Haemost 2001;85:771-4.

18. Mastora I, Remy-Jardin M, Masson P, Galland E, Delannoy $\mathrm{V}$, Bauchart JJ, et al. Severity of acute pulmonary embolism: evaluation of a new spiral CT angiographic score in correlation with echocardiographic data. Eur Radiol 2003;13:29-35.

19. Qanadli SD, El Hajjam M, Vieillard-Baron A, Joseph J, Mesurolle B, Oliva VL, et al. New CT index to quantify arterial obstruction in pulmonary embolism: comparison with angiographic index and echocardiography. AJR Am J Roentgenol 2001;176:1415-20.

20. Björgell O, Nilsson PE, Svensson PJ, Bergqvist D. A new scoring system for the detailed description of the distribution and thrombotic burden in deep leg vein thrombosis. Angiology 1999;50:179-87.

21. Ouriel K, Greenberg RK, Green RM, Massullo JM, Goines DR. A volumetric index for the quantification of deep venous thrombosis. J Vasc Surg 1999;30:1060-6.

22. Kistner RL, Ball JJ, Nordyke RA, Freeman GC. Incidence of pulmonary embolism in the course of thrombophlebitis of the lower extremities. Am J Surg 1972;124:169-76.

23. Martin F, Leroyer C, Oger E, Bressollette L, André N, Nonent M, et al. Pulmonary embolism and the level of thrombosis. A prospective study of 155 patients. Rev Mal Respir 1995;12:465-9.
24. Lusiani L, Visona A, Bonanome A, Pesavento R, Zanco P. The characteristics of the thrombi of the lower limbs, as detected by ultrasonic scanning, do not predict pulmonary embolism. Chest 1996;110:996-1000.

25. Meignan M, Rosso J, Gauthier H, Brunengo F, Claudel S, Sagnard L, et al. Systematic lung scans reveal a high frequency of silent pulmonary embolism in patients with proximal deep venous thrombosis. Arch Intern Med 2000;160:159-64.

26. Fink AM, Mayer W, Steiner A. Extent of thrombus evaluated in patients with recurrent and first deep vein thrombosis. J Vasc Surg 2002;36:357-60.

27. Simonneau G, Sors H, Charbonnier B, Page Y, Laaban JP, Azarian R, et al. A comparison of low-molecular-weight heparin with unfractionated heparin for acute pulmonary embolism. The THESEE Study Group. Tinzaparine ou Heparine Standard: Evaluations dans l'Embolie Pulmonaire. N Engl J Med 1997;337:663-9.

28. Björgell O, Nilsson PE, Benoni G, Bergqvist D. Symptomatic and asymptomatic deep vein thrombosis after total hip replacement. Differences in phlebographic pattern, described by a scoring of the thrombotic burden. Thromb Res 2000;99:429-38.

29. Bankier AA, Janata K, Fleischmann D. Severity assessment of acute pulmonary embolism with spiral CT: evaluation of two modified angiographic scores and comparison with clinical data. J Thor Imag 1997;12:150-8.

30. Collomb D, Paramelle PJ, Calaque O, Bosson JL, Vanzetto G, Barnoud D, et al. Severity assessment of acute pulmonary embolism: evaluation using helical CT. Eur Radiol 2003;13:1508-14.

31. Metafratzi ZM, Vassiliou MP, Maglaras GC, Katzioti FG, Constantopoulos SH, Katsaraki A, et al. Acute pulmonary embolism: correlation of CT pulmonary artery obstruction index with blood gas values. AJR Am J Roentgenol 2006;186:213-9.

32. Loud PA, Katz DS, Bruce DA, Klippenstein DL, Grossman ZD. Deep venous thrombosis with suspected pulmonary embolism: detection with combined CT venography and pulmonary angiography. Radiology 2001;219:498-502.

33. Remy-Jardin M, Remy J, Artaud D, Deschildre F, Duhamel A. Peripheral pulmonary arteries: optimization of the spiral CT acquisition protocol. Radiology 1997;204:157-63.

34. Wells PS, Anderson DR, Bormanis J. Value of assessment of pretest probability of deep-vein thrombosis in clinical management. Lancet 1997;350:1795-8.

35. Altman DG. Comparing groups - continuous data. In: Altman DG, editor. Practical statistics for medical research. London, UK: Chapman \& Hall/CRC, 1999: 210-2. 